\title{
THE EFFECT OF PLAYING FORMATION ON THE PASSING NETWORK CHARACTERISTICS OF A PROFESSIONAL FOOTBALL TEAM
}

original paper

(1) University School of Physical Education in Wroclaw

DOI: https://doi.org/10.5114/hm.2018.79416

\author{
SCOTT MCLEAN ${ }^{1}$, PAUL M. SALMON ${ }^{1}$, ADAM D. GORMAN ${ }^{2}$, JASON WICKHAM ${ }^{3}$, \\ ELISE BERBER ${ }^{1}$, COLIN SOLOMON ${ }^{1,2}$ \\ ${ }^{1}$ Centre for Human Factors and Sociotechnical Systems, University of the Sunshine Coast, Sippy Downs, Australia \\ ${ }^{2}$ School of Health and Sport Sciences, University of the Sunshine Coast, Sippy Downs, Australia \\ ${ }^{3}$ Brisbane Roar Football Club, Brisbane, Australia
}

\begin{abstract}
Purpose. The playing formation of football teams influences how players interact with each other via passing. The aim of this study was to use network analysis to determine the passing characteristics of playing positions within a professional a professional football team across two consecutive competitive seasons, when playing two different formations.

Methods. In season one (2016/2017) the team played 1-4-2-2-2 in 21 matches, and in season two (2017/2018) the team played 1-4-2-3-1 in 21 matches. Network analysis was applied to calculate the individual centrality metrics of indegree centrality (IDC), outdegree centrality (ODC), closeness centrality (CC), and betweenness centrality (BC) for the playing positions, using the Social Network Visualizer (SocNetV). The centrality metrics were compared across the playing formations and as a function of match outcome.
\end{abstract}

Results. The forward positions in 1-4-2-2-2 had significantly $(p<0.05)$ more outgoing passes compared with 1-4-2-3-1. The defensive midfield positions in 1-4-2-3-1 had a significantly $(p<0.05)$ higher \%BC compared with $1-4-2-2-2$. The forward positions had increased \%IDC and \%ODC when playing 1-4-2-2-2 in matches drawn compared with 1-4-2-3-1.

Conclusions. The current study shows that subtle changes to playing formation elicit differences in the passing contributions of the players. The results suggest that coaches may adopt the playing formation 1-4-2-2-2 compared with 1-4-2-3-1 owing to the increased passing involvement from the forwards.

Key words: network analysis, centrality, performance analysis, football

\section{Introduction}

The playing formation of a football team is critical for implementing the specific tactics and style of play that the coach wants to achieve [1]. As such, the playing formation and its influence on match performance and match outcome is generally accepted as one of the most discussed and debated topics in football. Playing formations in football are used to classify the team structure on the basis of groupings of playing positions [1]. One example among the numerous playing formations is the classic 1-4-4-2 formation, which consists of one goalkeeper, four defenders, four midfielders, and two forwards.
Previous research has shown that the use of different formations places different physical, technical, and tactical demands on players within the team [2-5]. In the English Premier League, the physical demands of players differ as a function of formation, with attackers in a 1-4-3-3 formation covering greater total, high, and very high intensity running distances compared with the 1-4-4-2 and 1-4-5-1 formations [3]. In contrast, defenders in a 1-4-4-2 formation cover increased total and high intensity running distances compared with defenders in the 1-4-3-3 and 1-4-5-1 formations [3]. For skill-related performance variables, a French Ligue 1 team performed more passes against a 1-4-2-2 compared with a 1-4-2-3-1

Correspondence address: Scott Mclean, University of the Sunshine Coast, 90 Sippy Downs Drive, Sippy Downs 4556, Australia, e-mail: smclean@usc.edu.au

Received: October 2, 2018

Accepted for publication: October 31, 2018

Citation: Mclean S, Salmon PM, Gorman AD, Wickham J, Berber E, Solomon C. The effect of playing formation on the passing network characteristics of a professional football team. Hum Mov. 2018;19(5)special/issue:14-22; doi: https://doi. org/10.5114/hm.2018.79416. 
formation, but not against the formations of 1-4-3-3 and 1-4-5-1 in 45 matches across three seasons [2]. These studies demonstrate the influence of formation on the physical and technical demands of players in matches.

Network analysis is a method of analysing the structural properties of teams via the interactions between players, and identifies individual players' passing behaviours within different formations [6]. Network analysis views the players as nodes in a network and the passes between players as the connections linking the nodes $[7,8]$. Network analysis has been applied in football match analyses to determine overall match passing characteristics [9], goal scoring passing networks [10, 11], and intra-team communication [12].

Within network analysis, centrality indices are commonly used to understand nodal importance [7, 8]. In football and other team sports where players are connected via passing, centrality metrics have been used to identify prominent players on the basis of their contribution to the passing networks [13]. The use of centrality indices has included indegree centrality (IDC), outdegree centrality (ODC), closeness centrality (CC), and betweenness centrality (BC) to determine nodal prominence [6, 14-16]. The IDC and ODC indices have been applied to quantify the connectivity of individual players to other players in terms of incoming and outgoing passes, respectively [6]. The CC index is used to identify well connected players within a team in terms of how easy it is to reach them (i.e. the shortest geodesic distance) $[14,15]$. The BC index identifies the players who connect other pairs of players, i.e. acting as a bridge between pairs of players [14, 15] (see 'Material and methods' for details of centrality indices).

One study investigated the effect of formation on the centrality metrics of playing positions in an international tournament. For the 2014 World Cup, the influence of four formations (1-4-3-3, 1-4-2-3-1, 1-4$4-2$, and $1-3-5-2)$ on the centrality metrics of playing positions was investigated [6]. The results indicated that irrespective of the formation used, the central midfielders and central defenders were typically the highest contributors to the team passing performances compared with the other positions [6,17]. These findings are useful for informing coaches of the passing contributions and tactical behaviours of players in specific playing formations. However, matches played per team at the World Cup are limited, as the maximum number of games a team can play is seven, and the minimum number of games is three. Therefore, a more detailed analysis of passing contributions of playing positions may be achieved by analysing professional teams across competitive seasons using different playing formations.

Therefore, the aim of this study was to apply centrality indices to determine the passing performances of playing positions of a professional football team playing two different formations across two consecutive competitive seasons.

\section{Material and methods}

\section{Study design}

The study was designed to compare the passing characteristics of the same professional football team across two consecutive seasons of the Australian A league (professional league in Australia). In season one (2016/ 2017), the team used a 1-4-2-2-2 formation (Figure 1) in 21 competitive league matches (seven wins, eight draws, six losses) with 7364 completed passes; in season two (2017/2018), the team used a 1-4-2-3-1 formation (Figure 1) in 21 competitive league matches (seven wins, five draws, nine losses) with 5722 completed passes. Centrality metrics for the individual playing positions were calculated for each game for the two seasons, and as a function of match outcome (win, draw, loss). The centrality metrics calculated for each playing position for each match were percentage outdegree centrality (\%ODC), percentage indegree centrality (\%IDC), percentage closeness centrality (\%CC), and percentage betweenness centrality (\%BC) [6]. Match passing data were provided by Opta Sports, which is a reliable system for the analysis of match actions [18]. For the analysis, individual playing positions were grouped to allow for a direct comparison between playing the two formations (Figure 1).

\section{Data analysis}

\section{Network analysis}

For each match within the two seasons, a network matrix was produced (example in Table 1) for the completed passes within a match $[7,8]$. The networks were directional (i.e. player A to player B) and weighted (i.e. denoting the number of passes) [10, 11]. In total, 42 (21 for each season) network matrices were produced to calculate the centrality metrics using the Social Network Visualizer (SocNetV). All centrality metrics applied in the current study are expressed as a percentage. 


\section{HUMAN MOVEMENT}

S. Mclean et al., The effect of playing formation on passing networks
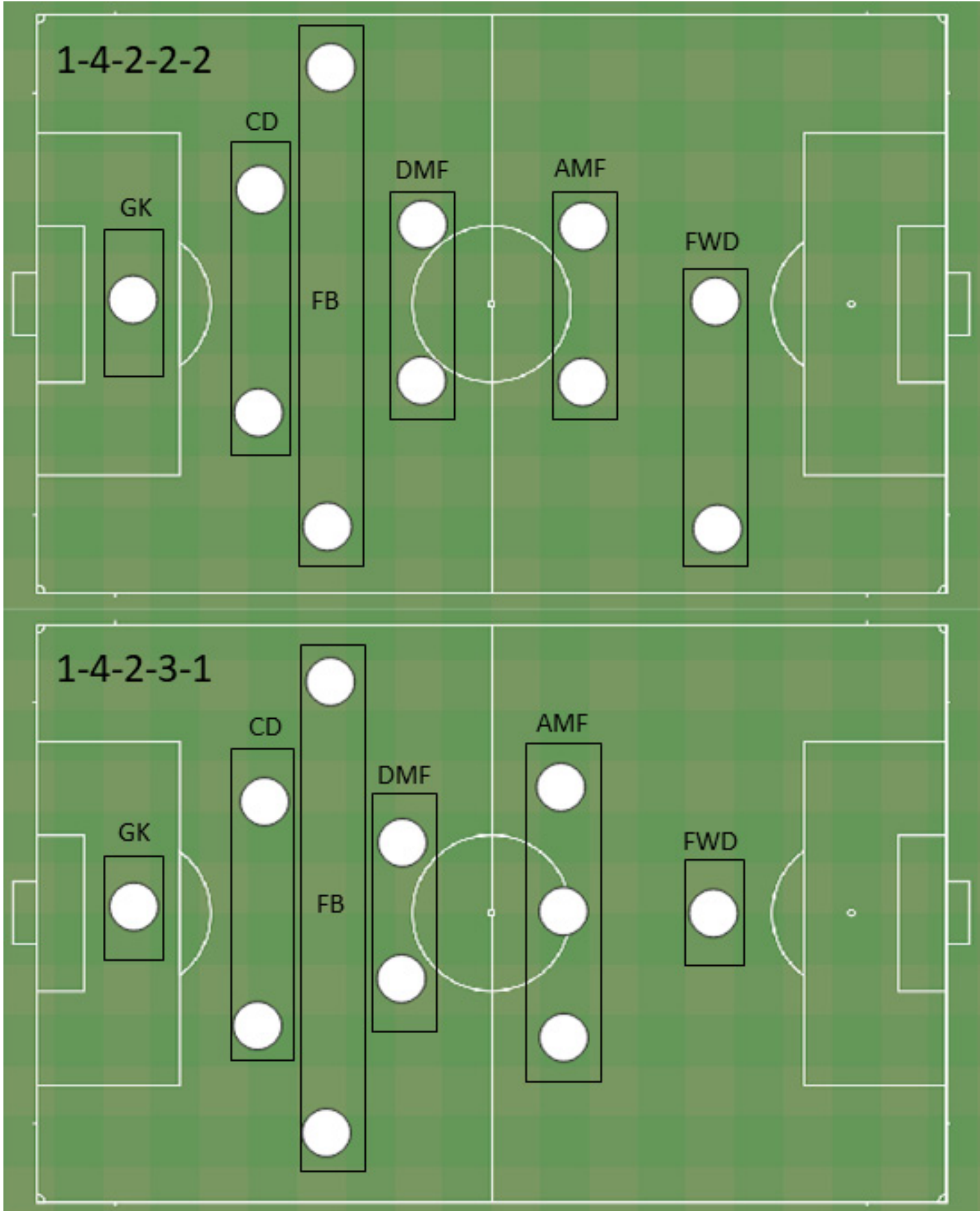

1-4-2-2-2:

GK - goalkeeper

$\mathrm{CD}$ - right and left central defenders

FB - right and left fullbacks

DMF - right and left defensive midfielders

AMF - right and left attacking midfielders, FWD - right and centre forwards

1-4-2-3-1:

GK - goalkeeper

FB - right and left fullbacks

$C D$ - right and left central defenders

DMF - right and left defensive midfielders

AMF - right, central, and left attacking midfielders

FWD - centre forward

The GK, CD, and FB make up the defensive line.

Figure 1. Playing formations for the two seasons investigated. The upper panel represents the formation played in season one (1-4-2-2-2). The lower panel represents the formation played in season two (1-4-2-3-1).

Boxes indicate position groupings. Direction of attack is left to right 
Table 1. Example passing social network matrix for a match

\begin{tabular}{cccccccccccc}
\hline Player & 1 & 2 & 3 & 4 & 5 & 6 & 7 & 8 & 9 & 10 & 11 \\
\hline 1 & & 4 & 7 & 2 & 4 & 0 & 0 & 0 & 0 & 0 & 0 \\
2 & 0 & & 8 & 1 & 0 & 5 & 4 & 4 & 4 & 15 & 0 \\
3 & 2 & 9 & & 18 & 1 & 7 & 7 & 3 & 1 & 3 & 1 \\
4 & 2 & 2 & 19 & & 15 & 3 & 9 & 0 & 3 & 0 & 1 \\
5 & 0 & 0 & 0 & 15 & & 3 & 8 & 1 & 5 & 4 & 1 \\
6 & 1 & 4 & 9 & 3 & 2 & & 8 & 4 & 4 & 3 & 0 \\
7 & 1 & 5 & 3 & 10 & 10 & 9 & & 6 & 11 & 5 & 2 \\
8 & 0 & 4 & 1 & 0 & 2 & 2 & 6 & & 8 & 5 & 0 \\
9 & 0 & 2 & 1 & 2 & 4 & 3 & 5 & 4 & & 3 & 3 \\
10 & 0 & 5 & 0 & 0 & 0 & 1 & 1 & 7 & 0 & & 1 \\
11 & 0 & 0 & 0 & 0 & 0 & 1 & 1 & 0 & 4 & 0 & \\
\hline
\end{tabular}

Data are the absolute numbers of passes between players (e.g. player 1 passed to player 2 on four occasions, to player 3 on seven occasions).

\section{Percentage outdegree centrality $(\% O D C)$}

The ODC index is a measure of the activity of a player in terms of passing, and quantifies the number of links (passes) that a node (player) has to other nodes within the network [6]. In directed graphs, the ODC is the total number of passes out of a node to all other nodes. In the football context, players who have high values for ODC contribute more to the teams' passing network compared with players with lower ODC values.

\section{Percentage indegree centrality (\%IDC)}

The IDC index is a measure of the activity of a player in terms of received passes, and quantifies the number of ties (passes) that a node (player) receives from other nodes within the network [6]. In the football context, players who have high values for IDC are those who receive more passes from all other players compared with players with lower IDC values.

\section{Percentage closeness centrality (\%CC)}

The CC index quantifies nodal importance in terms of geodesic distance within a social network $[14,15]$. In graph theory, the geodesic distance of two nodes is the minimum number of edges required to connect the two nodes $[14,15]$. The CC index considers how close each node is to all other nodes in the network. In the football context, players with high CC values are those who reach many other players in a few passes (i.e. a player is more central and has a high CC value if they can interact with more of the other players).

\section{Percentage betweenness centrality $(\% B C)$}

The BC index is a measure of the extent to which a node (player) lies on a path between two nodes, i.e. the number of times when a player acts as a bridge in the shortest path connecting pairs of nodes [6, 19]. The $\mathrm{BC}$ is not a measure of how well connected a player is, rather of how the ball flows through this position and connects players. In the football context, a player with a high $\mathrm{BC}$ connects a high number of players.

\section{Statistical analysis}

The centrality metric values for the playing positions were calculated in SocNetV. Statistical analyses were performed with SPSS (version 24, IBM Corporation, Armonk, NY, USA). One-way analysis of variance tests (ANOVA) were performed to determine differences between the playing positions as a function of playing formation across the 21 matches, and for the comparisons of playing positions across playing formations as a function of match outcome. The level of significance was set at $\alpha=0.05$, and the statistical power $(\beta)$ was reported. Partial eta squared $\left(\mathrm{n}_{\mathrm{P}}{ }^{2}\right)$ was calculated as an indicator of effect size, with values defined as small (0.01), medium (0.06), and large (0.14) [20]. The calculation for partial eta squared was the sum of squares (SS) effect / SS effect + SS error. Levene's test for homogeneity of variance was performed for all ANOVAs, with no significance $(p>0.05)$ found.

\section{Ethical approval}

The research related to human use has been complied with all the relevant national regulations and institutional policies, has followed the tenets of the 
Declaration of Helsinki, and has been approved by the Human Research Ethics Committee of the University of the Sunshine Coast (E18101).

\section{Results}

\section{Overall season}

For the overall season match comparison $(n=21)$, there was a significant increase and medium effect size between the forward (FWD) positions $\left(p=0.030 ;{n_{P}}^{2}=\right.$ $0.112 ; \beta=0.592)$ for $\% O D C$ in the $1-4-2-2-2$ compared with the 1-4-2-3-1 formation, and a significant increase and medium effect size between the defensive midfielder (DMF) positions $\left(p=0.044 ; \eta_{\mathrm{P}}^{2}=0.097 ; \beta=\right.$ 0.527 ) for $\% \mathrm{BC}$ in the 1-4-2-3-1 compared with the 1-4-2-2-2 formation (Figure 2). There were no significant $(p<0.05)$ differences between the playing positions for \%IDC or \%CC as a function of playing formation.

\section{Match outcome}

For \%ODC, there were significant increases and large effect sizes for the DMF $\left(p=0.044 ; \eta_{\mathrm{P}}{ }^{2}=0.297\right.$; $\beta=0.544)$ in matches won, and for the FWD ( $p=0.006$; $\mathrm{n}_{\mathrm{p}}{ }^{2}=0.508 ; \beta=0.866$ ) in matches drawn as a function of playing formation (Table 2). For \%IDC, there was a significant increase and large effect size for the FWD $\left(p=0.001 ; n_{P}^{2}=0.660 ; \beta=0.987\right)$ in matches drawn as a function of playing formation (Table 2). There were no significant differences $(p<0.05)$ between playing positions as a function of match outcome for $\% \mathrm{CC}$ or \%BC (Table 2).

\section{Discussion}

For the current study, centrality indices were used to measure the passing contributions of playing positions within a professional football team across two different playing formations. Overall, the change of formation between the two seasons had little influence on the overall passing contributions to the team but there were significant changes in the connectivity of forward and defensive midfield positions. The results have important practical implications for coaches.

In the comparison of the 21 games played with two different formations, the significant difference between the FWD positions for \%ODC indicates that outgoing passes in the FWD positions were increased when
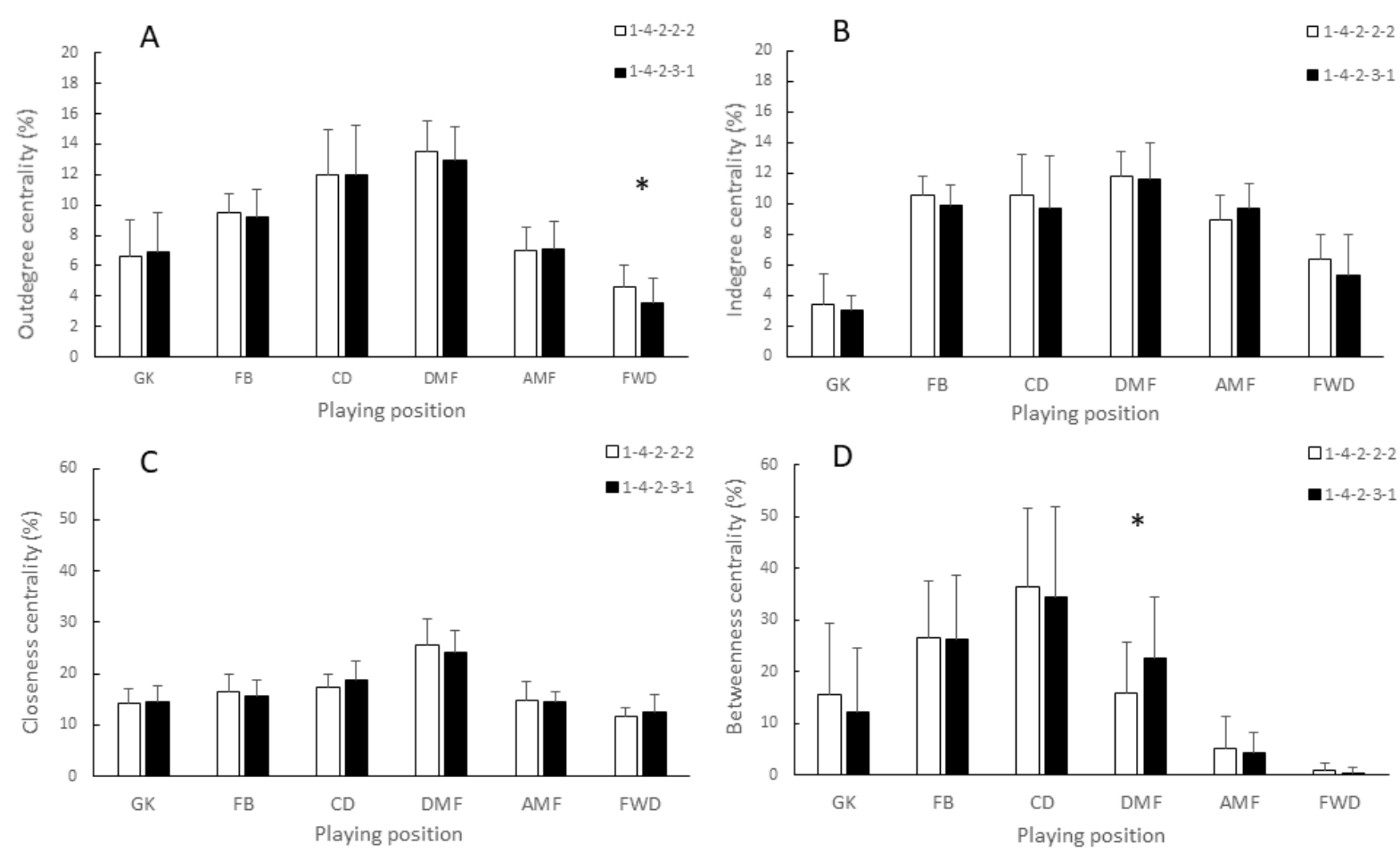

GK - goalkeepers, CD - central defenders, FB - fullbacks, DMF - defensive midfielders, AMF - attacking midfielders, FWD - forwards

* significant differences $(p>0.05)$

Figure 2. Comparison of centrality metric values of playing positions between playing formations 1-4-2-2-2 and 1-4-2-3-1. Outdegree centrality (panel A), indegree centrality (panel B), closeness centrality (panel C), and betweenness centrality (panel D). Data are means and $S D$ 
Table 2. Positional values for outdegree centrality (\%ODC), indegree centrality (\%IDC), closeness centrality (\%CC), and betweenness centrality (\%BC) for the two formations between match outcomes

\begin{tabular}{|c|c|c|c|c|c|c|c|}
\hline Wins & Formation & GK & FB & $\mathrm{CD}$ & DMF & $\mathrm{AMF}$ & FWD \\
\hline \multirow{2}{*}{$\% \mathrm{ODC}$} & $1-4-2-2-2$ & $6.1 \pm 1.7$ & $9.2 \pm 1.2$ & $10.3 \pm 1.7$ & $15.0 \pm 1.0^{*}$ & $7.7 \pm 0.9$ & $4.8 \pm 1.4$ \\
\hline & $1-4-2-3-1$ & $6.3 \pm 2.4$ & $9.1 \pm 2.0$ & $10.5 \pm 2.8$ & $12.7 \pm 2.5$ & $8.1 \pm 1.8$ & $4.8 \pm 1.9$ \\
\hline \multirow{2}{*}{$\%$ IDC } & $1-4-2-2-2$ & $3.5 \pm 1.0$ & $10.6 \pm 1.1$ & $8.9 \pm 2.0$ & $12.4 \pm 1.6$ & $10.0 \pm 1.1$ & $6.3 \pm 0.9$ \\
\hline & $1-4-2-3-1$ & $2.9 \pm 1.4$ & $10.1 \pm 1.2$ & $8.2 \pm 3.2$ & $10.8 \pm 3.2$ & $10.8 \pm 1.4$ & $6.6 \pm 3.9$ \\
\hline \multirow{2}{*}{$\% \mathrm{CC}$} & $1-4-2-2-2$ & $14.5 \pm 3.3$ & $14.6 \pm 4.2$ & $15.7 \pm 1.8$ & $28.0 \pm 6.1$ & $15.5 \pm 3.1$ & $11.7 \pm 1.8$ \\
\hline & $1-4-2-3-1$ & $13.7 \pm 3.1$ & $16.4 \pm 3.4$ & $18.9 \pm 3.9$ & $23.7 \pm 4.1$ & $15.1 \pm 1.0$ & $12.1 \pm 4.6$ \\
\hline \multirow{2}{*}{$\% \mathrm{BC}$} & $1-4-2-2-2$ & $19.8 \pm 8.7$ & $26.2 \pm 12.9$ & $28.4 \pm 10.1$ & $18.5 \pm 10.4$ & $6.8 \pm 7.6$ & $0.3 \pm 0.4$ \\
\hline & 1-4-2-3-1 & $11.1 \pm 15.5$ & $28.1 \pm 12.2$ & $23.9 \pm 13.1$ & $27.6 \pm 12.1$ & $8.4 \pm 3.8$ & $0.9 \pm 1.7$ \\
\hline Draws & Formation & GK & $\mathrm{FB}$ & $\mathrm{CD}$ & DMF & $\mathrm{AMF}$ & FWD \\
\hline \multirow{2}{*}{$\% \mathrm{ODC}$} & $1-4-2-2-2$ & $7.3 \pm 3.5$ & $9.7 \pm 1.3$ & $12.4 \pm 3.8$ & $12.8 \pm 2.3$ & $6.5 \pm 2.1$ & $5.0 \pm 1.4^{*}$ \\
\hline & $1-4-2-3-1$ & $7.0 \pm 3.4$ & $9.7 \pm 2.1$ & $12.5 \pm 4.9$ & $13.0 \pm 3.2$ & $6.6 \pm 2.4$ & $2.7 \pm 0.8$ \\
\hline \multirow{2}{*}{ \%IDC } & $1-4-2-2-2$ & $3.7 \pm 3.1$ & $10.7 \pm 1.2$ & $10.9 \pm 3.0$ & $11.1 \pm 1.7$ & $8.2 \pm 1.9$ & $7.3 \pm 1.2^{*}$ \\
\hline & $1-4-2-3-1$ & $3.0 \pm 0.6$ & $10.1 \pm 1.4$ & $11.3 \pm 5.2$ & $11.6 \pm 2.9$ & $9.0 \pm 1.9$ & $4.1 \pm 1.2$ \\
\hline \multirow{2}{*}{$\% \mathrm{CC}$} & $1-4-2-2-2$ & $14.7 \pm 3.3$ & $17.8 \pm 3.0$ & $18.7 \pm 2.5$ & $24.0 \pm 4.0$ & $13.4 \pm 4.0$ & $11.4 \pm 1.0$ \\
\hline & $1-4-2-3-1$ & $14.8 \pm 1.6$ & $16.8 \pm 3.3$ & $19.2 \pm 4.0$ & $23.4 \pm 3.3$ & $14.6 \pm 1.9$ & $11.2 \pm 2.9$ \\
\hline \multirow{2}{*}{$\% \mathrm{BC}$} & $1-4-2-2-2$ & $11.3 \pm 11.9$ & $23.5 \pm 7.9$ & $43.3 \pm 18.2$ & $14.7 \pm 8.1$ & $5.5 \pm 7.4$ & $1.6 \pm 2.0$ \\
\hline & $1-4-2-3-1$ & $11.4 \pm 13.9$ & $26.8 \pm 13.6$ & $39.7 \pm 15.7$ & $19.5 \pm 11.8$ & $2.5 \pm 2.2$ & $0.0 \pm 0.0$ \\
\hline Losses & Formation & GK & $\mathrm{FB}$ & $\mathrm{CD}$ & DMF & $\mathrm{AMF}$ & FWD \\
\hline \multirow{2}{*}{$\% \mathrm{ODC}$} & $1-4-2-2-2$ & $6.2 \pm 1.3$ & $9.8 \pm 1.2$ & $13.5 \pm 2.0$ & $12.8 \pm 1.7$ & $7.0 \pm 1.0$ & $4.0 \pm 1.3$ \\
\hline & $1-4-2-3-1$ & $7.4 \pm 2.4$ & $8.9 \pm 1.9$ & $12.8 \pm 2.5$ & $13.1 \pm 1.5$ & $6.6 \pm 1.1$ & $3.1 \pm 1.2$ \\
\hline \multirow{2}{*}{$\%$ IDC } & $1-4-2-2-2$ & $3.2 \pm 1.1$ & $10.4 \pm 1.6$ & $11.9 \pm 2.5$ & $12.1 \pm 1.5$ & $8.7 \pm 1.1$ & $5.3 \pm 2.0$ \\
\hline & $1-4-2-3-1$ & $3.1 \pm 1.0$ & $9.8 \pm 1.3$ & $9.9 \pm 2.1$ & $12.3 \pm 1.6$ & $9.4 \pm 1.3$ & $5.0 \pm 1.8$ \\
\hline \multirow{2}{*}{$\%$ CC } & $1-4-2-2-2$ & $13.6 \pm 1.6$ & $16.8 \pm 2.4$ & $17.4 \pm 2.6$ & $24.9 \pm 5.2$ & $15.5 \pm 4.2$ & $11.7 \pm 2.9$ \\
\hline & $1-4-2-3-1$ & $15.0 \pm 3.8$ & $14.6 \pm 2.5$ & $18.3 \pm 4.0$ & $24.8 \pm 5.5$ & $14.1 \pm 2.3$ & $13.3 \pm 3.2$ \\
\hline \multirow{2}{*}{$\% \mathrm{BC}$} & $1-4-2-2-2$ & $16.6 \pm 20.1$ & $30.5 \pm 13.3$ & $36.2 \pm 13.2$ & $13.7 \pm 12.4$ & $2.4 \pm 2.5$ & $0.5 \pm 0.9$ \\
\hline & $1-4-2-3-1$ & $13.4 \pm 10.2$ & $24.5 \pm 13.0$ & $39.3 \pm 19.8$ & $20.6 \pm 11.5$ & $2.2 \pm 1.3$ & $0.0 \pm 0.0$ \\
\hline
\end{tabular}

GK - goalkeepers, FB - fullbacks, CD - central defenders, DMF - defensive midfielders, AMF - attacking midfielders,

FWD - forwards

* significant difference between 1-4-2-2-2 and 1-4-2-3-1 $(p<0.05)$

Data are means $\pm S D$.

playing with two FWDs in 1-4-2-2-2 compared with a single FWD in 1-4-2-3-1. The most likely explanation for this difference is the outgoing passing contribution of the additional FWD in the 1-4-2-2-2 formation. In other words, the formation that involved two FWDs had more outgoing passes from these players compared with the formation with only a single FWD.

The current results for \%ODC are similar to the contribution of the FWDs in the 2014 World Cup [6], whereby the \%ODC of FWDs in a formation with two FWDs (1-3-5-2) was higher than with a single FWD (1-4-2-3-1). This finding has practical implications for coaches by highlighting that playing with two FWDs may enable increased ball possession in the opposition half, as this is generally where the FWDs are tactically positioned when in possession. Importantly, the increased passing contribution of the FWDs in 1-4-2-2-2 compared with 1-4-2-3-1 occurred without changes to the passing structures for any other playing positions across the two playing formations. Therefore, coaches may choose to play with a 1-4-2-2-2 compared with a 1-4-2-3-1 formation because of the increased passing involvement of the FWDs without effecting the outgoing passing contributions of the other 
playing positions. From a defensive perspective, increased passing contribution for the FWDs in a 1-42-2-2 formation could indicate that there is reduced time of play in the team's defensive half. This is important for coaches to consider as goals are regularly scored from turnovers of possession within a team's own half $[10,11]$, and so increased possession in the opposition half potentially reduces the threat of turnovers of possession in a team's own half.

The significant increase in \%BC for the DMF in 1-4-2-3-1 suggests that the two DMF players were able to connect other pairs of players more often compared with the 1-4-2-2-2 formation. A potential explanation for the increase in $\% \mathrm{BC}$ is that the DMFs were able to connect the defence and attacking midfielders (AMF) more often in the 1-4-2-3-1 formation owing to the additional player in the AMF line compared with 1-4-2-2-2. This is indicated by the higher \%IDC for the AMF when playing 1-4-2-3-1 compared with 1-4-2-2-2 (Figure 2).

Despite only three significant results for the effect of playing formation on match outcome, there were changes to the players who would be expected to be influenced by the different formations. In this case, the FWD positions in 1-4-2-2-2 contributed more \%ODC and \%IDC in matches drawn compared with 1-4-2-3-1. From a practical perspective, this finding has important implications for coaches because it highlights how even quite subtle changes to a formation can elicit different passing contributions from certain playing positions. Given that the defensive structure of four defenders and two DMFs was the same in both formations in this study, an interesting comparison for future research would be to analyse a formation using three defenders across a season. This may reveal differences in the passing contributions of the playing positions and extend the knowledge of a teams' season-long passing structure with fewer defenders.

The current study did not compare the centrality metrics between positions within the formations as these have previously been shown to be different $[6,14]$. Furthermore, these differences could be expected as a result of the high volume of passing in football being centred around the midfielders (MF) and central defenders (CD).

The presented study has demonstrated the use of highly descriptive centrality metrics for the analysis of passing contributions of playing positions in football. By identifying the prominent passers and receivers, as well as the positions regularly interacting with others and the positions that play an intermediary role in connecting players, the use of centrality metrics enables the classification of a team's style of play. This has implications for post-match assessment of passing, as well as for opposition passing analysis [11]. The current study has shown that relatively subtle changes in playing formation can elicit important practical changes in the playing styles of key players (FWDs in the case of the present research). The high centrality values among the CD and DMF players indicate that the current team's style is based on high ball possession and build-up from defence. These results support previous research in which central MFs and CDs were the most prominent passers at the 2014 World Cup, displaying higher ODC and IDC values compared with the other positions $[6,17]$.

In 2010, Spain, the eventual winners of the World Cup were characterized by evenly distributed values of $\mathrm{CC}$ and low values of $\mathrm{BC}$ across the team i.e. not reliant on a few key players [14]. Evenly distributed CC values and low $\mathrm{BC}$ values for a team's passing performance indicate a balanced team passing structure that is not necessarily reliant on a small number of players passing contributions [15]. In consideration of these passing characteristics, the passing strategies of the team in this study appear to be inconsistent with the balanced passing structure described in previous research. In the current study, the DMFs had high values for CC, indicating that these positions continually interacted with multiple team members compared with the other playing positions. Furthermore, the high $\mathrm{BC}$ values for the CDs across both playing formations indicate a heavy reliance on these positions to be the intermediary players in connecting others. A negative aspect of the current team's passing structure is that it may be predictable to the opposition. For coaches adopting a possession-based style of play, the centrality metrics demonstrated here could be applied to objectively determine whether the desired passing structure is being achieved. However, it should be noted that the recommendations of optimal passing characteristics indicated in this study might apply to teams wishing to play with high possession and a balanced passing style, whereas in reality, teams can be effective by playing alternative styles of play which will display different passing characteristics.

\section{Limitations}

The current study contained expected limitations. Firstly, the opposition playing formation was not considered in the analysis; it is reasonable to assume that the structure of the opposition influenced the passing structure of the team in the study. However, the analysis was conducted over 21 competitive matches in each 
season in which the opponents played several different formations. Secondly, the grouping of positions may have reduced the influence of individual positions, although a direct comparison between formations would not have been possible without positional grouping. Thirdly, network analysis is a static analysis of passing, so more comprehensive passing analyses could be achieved by making the network analysis dynamic in future research.

\section{Conclusions}

In conclusion, the change of formation from 1-4-2-2-2 to 1-4-2-3-1 across consecutive seasons had minimal effect on the overall passing contributions of the team, but instead had a significant impact upon those players who were directly influenced by the formation changes. Specifically, the FWD positions in the 1-4-2-2-2 formation were able to contribute more outgoing passes compared with the 1-4-2-3-1 formation without any change to the other positions' passing contributions. This could encourage coaches to adopt the 1-4-2-2-2 over a 1-4-2-3-1 formation. Lastly, the study has extended the use of centrality metrics for determining the passing contributions across different formations, and as a method to indicate playing styles of football teams.

\section{Disclosure statement}

Paul Salmon's contribution to this research was supported by his current ARC Future Fellowship (FT140100681).

\section{Conflict of interest}

The authors state no conflict of interest.

\section{References}

1. Bangsbo J, Peitersen B. Soccer systems and strategies. Champaign: Human Kinetics; 2000.

2. Carling C. Influence of opposition team formation on physical and skill-related performance in a professional soccer team. Eur J Sport Sci. 2011;11(3):155-164; doi: 10.1080/17461391.2010.499972.

3. Bradley PS, Carling C, Archer D, Roberts J, Dodds A, Di Mascio M, et al. The effect of playing formation on high-intensity running and technical profiles in English FA Premier League soccer matches. J Sports Sci. 2011; 29(8):821-830; doi: 10.1080/02640414.2011.561868.

4. Clemente FM, Martins FML, Kalamaras D, Wong PD, Mendes RS. General network analysis of national soccer teams in FIFA World Cup 2014. Int J Perform Anal Sport. 2015;15(1):80-96; doi: 10.1080/24748668.2015. 11868778.
5. Tierney PJ, Young A, Clarke ND, Duncan MJ. Match play demands of 11 versus 11 professional football using Global Positioning System tracking: variations across common playing formations. Hum Mov Sci. 2016;49: 1-8; doi: 10.1016/j.humov.2016.05.007.

6. Clemente FM, Martins FML, Wong PD, Kalamaras D, Mendes RS. Midfielder as the prominent participant in the building attack: a network analysis of national teams in FIFA World Cup 2014. Int J Perform Anal Sport. 2015;15(2):704-722; doi: 10.1080/24748668.2015. 11868825.

7. Lusher D, Robins G, Kremer P. The application of social network analysis to team sports. Meas Phys Educ Exerc Sci.2010;14(4):211-224; doi:10.1080/1091367X.2010. 495559.

8. Wäsche H, Dickson G, Woll A, Brandes U. Social network analysis in sport research: an emerging paradigm. Eur J Sport Soc. 2017;14(2):138-165; doi: 10.1080/ 16138171.2017.1318198.

9. Grund TU. Network structure and team performance: the case of English Premier League soccer teams. Soc Networks. 2012;34(4):682-690; doi: 10.1016/j.socnet. 2012.08.004.

10. McLean S, Salmon PM, Gorman AD, Naughton M, Solomon C. Do inter-continental playing styles exist? Using social network analysis to compare goals from the 2016 EURO and COPA football tournaments knockout stages. Theor Issues Ergon Sci. 2017;18(4):370383; doi: 10.1080/1463922X.2017.1290158.

11. Mclean S, Salmon PM, Gorman AD, Stevens NJ, Solomon C. A social network analysis of the goal scoring passing networks of the 2016 European Football Championships. Hum Mov Sci. 2018;57:400-408; doi: 10.1016/ j.humov.2017.10.001.

12. Mclean S, Salmon PM, Gorman AD, Dodd K, Solomon C. Integrating communication and passing networks in football using social network analysis. Sci Med Football.2018:1-7; doi:10.1080/24733938.2018.1478122.

13. Sarmento H, Clemente FM, Araújo D, Davids K, McRobert A, Figueiredo A. What performance analysts need to know about research trends in association football (2012-2016): a systematic review. Sports Med. 2018; 48(4):799-836; doi: 10.1007/s40279-017-0836-6.

14. Peña JL, Touchette H. A network theory analysis of football strategies. arXiv:1206.6904. 2012.

15. Gonçalves B, Coutinho D, Santos S, Lago-Penas C, Jiménez S, Sampaio J. Exploring team passing networks and player movement dynamics in youth association football. PLoS One. 2017;12(1):e0171156; doi: 10.1371/ journal.pone.0171156.

16. Arriaza-Ardiles E, Martín-González JM, Zuniga MD, Sánchez-Flores J, de Saa Y, García-Manso JM. Applying graphs and complex networks to football metric interpretation. Hum Mov Sci. 2018;57:236-243; doi: 10.1016/j.humov.2017.08.022.

17. Clemente FM, Couceiro MS, Martins FML, Mendes RS. Using network metrics to investigate football team play- 


\section{HUMAN MOVEMENT}

S. Mclean et al., The effect of playing formation on passing networks

ers' connections: a pilot study. Motriz: Rev Educ Fis. 2014; 20(3):262-271; doi:10.1590/S1980-65742014000300004.

18. Liu H, Hopkins W, Gómez AM, Molinuevo SJ. Interoperator reliability of live football match statistics from OPTA Sportsdata. Int J Perform Anal Sport. 2013;13(3): 803-821; doi: 10.1080/24748668.2013.11868690.

19. Ribeiro J, Silva P, Duarte R, Davids K, Garganta J. Team sports performance analysed through the lens of social network theory: implications for research and practice. Sports Med. 2017;47(9):1689-1696; doi: 10.1007/ s40279-017-0695-1.

20. Levine TR, Hullett CR. Eta squared, partial eta squared, and misreporting of effect size in communication research. Hum Commun Res. 2002;28(4):612-625; doi: 10.1111/j.1468-2958.2002.tb00828.x. 